\title{
Extreme absorption in the bulk of sapphire with femtosecond Bessel pulses
}

\author{
Kazem Ardaneh, Remi Meyer, Benoit Morel, Remo Giust, Francois Courvoisier \\ FEMTO-ST Institute, Université Franche-Comté,UMR CNRS 6174,15B avenue des Montboucons, 25030 \\ Besançon, France \\ kazem.arrdaneh@gmail.com
}

\begin{abstract}
High absorption is experimentally measured during the propagation of femtosecond Bessel pulses in the bulk of sapphire. Using self-consistent particle-in-cell simulations, we have explained this process by resonance absorption in the pulse generated nanoscale plasma rod. (C) 2020 The Author(s)
\end{abstract}

\section{Introduction}

Ultrashort pulses are useful tools for the generation of a high-temperature, dense, and x-ray-emitting plasma at a solid's surface. The presence of dense plasmas is crucial in plasma mirrors, particle acceleration, x-ray lasers, and laser processing. For short pulses, the energy deposition occurs in the skin depth resulting in a fast ionization of the solid before significant ablation. Inside the bulk of the dielectric, however, several mechanisms, e.g., Kerr effect, nonlinear ionization, and defocussing, might seriously restrict the intensity reachable with spatially Gaussian pulses. High angle Bessel pulses behavior differently. They are generated by beam shaping from the cylindricallysymmetric interference of plane waves and their central high-intensity lobe can extend orders of magnitude larger than the Rayleigh length. Single-shot Bessel pulses have been used to create high aspect ratio channels and voids in transparent solid dielectrics, e.g., sapphire, fused silica, and glass [1,2]. In these experiments, an absorption of $\sim 50$ percent of pulse energy was measured. The radii of the concentric lobes, however, were unaffected which constrains the radius of the generated plasma inside the bulk $\lesssim 500 \mathrm{~nm}$.

In this work, we simulate the propagation of the femtosecond Bessel pulses into the transparent dielectric from the first principle using the Particle-In-Cell (PIC) technique. In this method, the trajectories of the individual particles are tracked in continuous phase-space while the electromagnetic fields are updated on a stationary grid using moments of the distribution, i. e., densities and currents, computed from the weighting of particles. The simulation results show that the resonance absorption in an overcritical nanoscale plasma can explain the extreme absorption in our experiments. Furthermore, it can reproduce the near-field fluence and far-field intensity pattern of experiments.

\section{Simulation setup}

We use Particle-In-Cell (PIC) code EPOCH [3]. We modeled the interaction between a $x$-polarized BesselGauss pulse with a pre-formed overcritical plasma rod. The central wavelength of the pulse and cone angle are $\lambda_{0}=0.8 \mu \mathrm{m}, \theta=25^{\circ}$, respectively. The pulse duration is $100 \mathrm{fs}$ Full Width at Half Maximum (FWHM). The Gaussian beam waist is $w_{0}=10 \mu \mathrm{m}$. The Gaussian pulse energy is $1.2 \mu \mathrm{J}$. We use an elliptical plasma rod elongated perpendicular to the laser polarization. The axial density distribution of the plasma rod is invariant density while the distribution in the $x y$-plane is Gaussian with an FWHM of $600 \mathrm{~nm}$ along the major axis and $250 \mathrm{~nm}$ along the minor axis. The plasma species initially have a Maxwellian distribution and are in equilibrium. The simulations are performed with and without collisions. We utilize a computational box of $15 \times 15 \times 30 \mu \mathrm{m}^{3}$ and run the simulations up to a time of $t_{\text {run }}=320 \mathrm{fs}$. The spatial resolution of the grid resolves the finest structure in the simulation with 20 cells. The temporal resolution is constrained by the Courant stability condition.

\section{Results and comparison with experiments}

In Figure 1, one can see a direct comparison between PIC results and the experimental measurements. The near field fluence distribution from the simulation is similar to the experimental one (top-right panel). The far-field radiation pattern from the simulation includes two lobes parallel to the laser polarization (bottom-middle panel) that is well-matched with the experimental one (bottom-right panel). From the simulations, we measured a very high absorption factor of $\sim 50 \%$ that is in agreement with values measured in experiments (top-left panel).

The fluence distribution around the plasma rod consists of two high-intensity lobes which are parallel to the laser polarization for the linear polarization (top-middle panel). This field amplification originates from the difference 

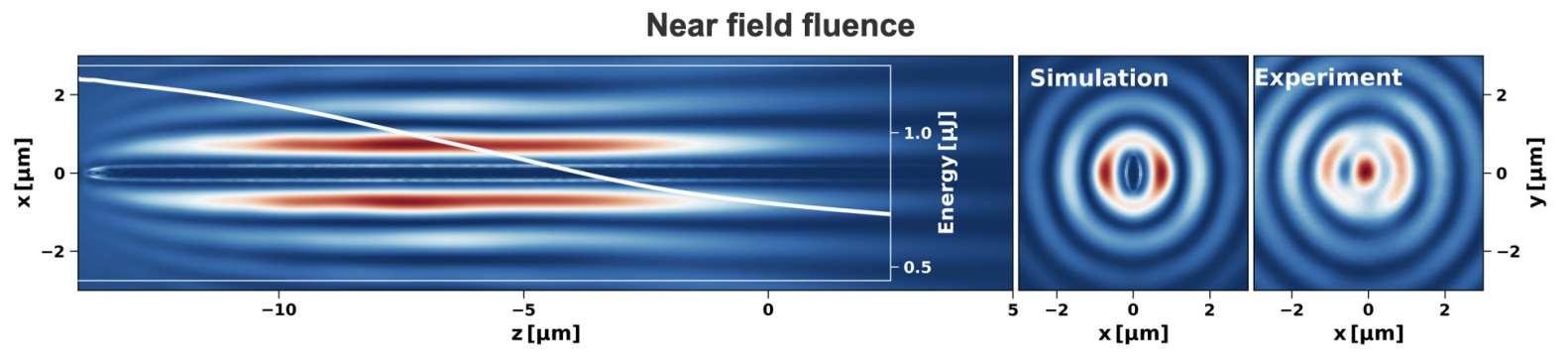

Plasma microphysics

Far field intensity

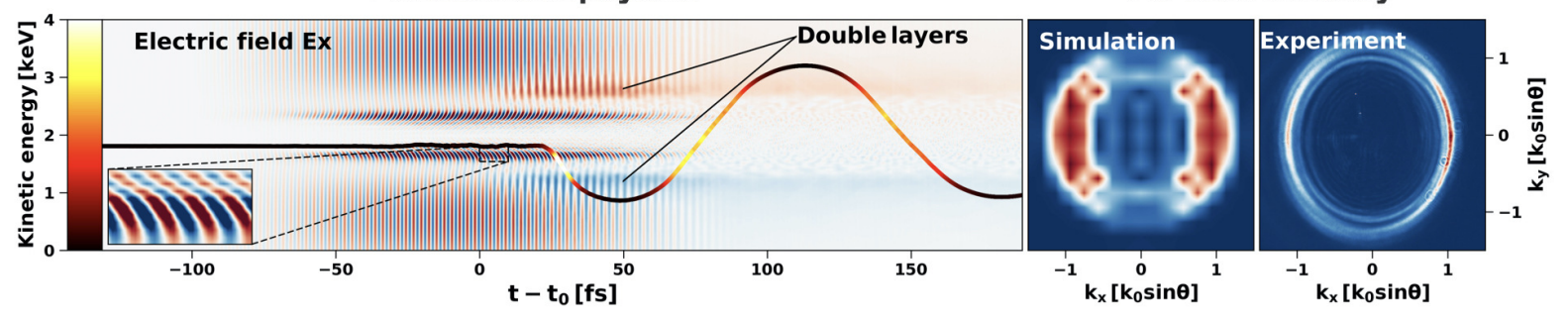

Fig. 1. Top row: Near-field fluence in the $z x$-plane (left), and $x y$-plane (middle) from the simulation, and near-field fluence in the $x y$-plane (right) from the experiment. The over-plotted line in the top-left shows the pulse energy versus the propagation distance. Bottom row: The trajectory of a representative particle over-plotted on the $E_{\mathrm{x}}$ electric field (left), the far-field intensity from the simulation (middle), and far-field intensity from the experiment (right). The color in the particle trajectory shoes the particle energy.

in permittivity between the plasma rod and the surrounding medium. The field amplification inside the plasma is due to the resonance absorption that happens for an obliquely incident $p$-polarized laser pulse on an overcritical plasma ramp [4]. The high absorption coefficient is indeed due to resonance absorption.

We have investigated the mechanism of particle energization by tracking the most energetic particles. The process of electron acceleration is due to the particle-wave interactions. We have noticed that particles are effectively accelerated via the wave-breaking process as they move into the high-intensity field with proper phase (bottom left panel). Their sharp energy increase in the particle energy follows a sharp decrease in the electric field energy. The hot electron population expands outwards. The double layers formed at the surface of the plasma due to the pressure gradient reflect most of the hot electrons while the energetic ones, those with energy beyond the potential of the double layer, leave the plasma volume. The radii of the trajectories increase with the time that is a characteristic behavior of the plasma expansion. The speed of this expansion is roughly the sound speed inside the plasma.

\section{Conclusion}

The propagation-invariant of Bessel pulses is the key for the homogeneous energy deposition over extended distances because the conical energy flow continuously compensates for the energy loss due to nonlinear ionization in the central lobe. Our simulations from the first principles show that resonance absorption happens in the bulk of the material due to the nanoscale plasma generation inside the bulk.

\section{References}

1. M. K. Bhuyan, F. Courvoisier, P. A. Lacourt, M. Jacquot, R. Salut, L. Furfaro, J. M Dudley, "High aspect ratio nanochannel machining using single shot femtosecond Bessel beams," Appl. Phys. Lett 97, 081102 (2010).

2. L. Froehly, M. Jacquot, P. A. Lacourt, J. M. Dudley, F. Courvoisier, "Spatiotemporal structure of femtosecond Bessel beams from spatial light modulators," J. Opt. Soc. Am. A 31, 790-793 (2014).

3. T. D. Arber, K. Bennett, C. S. Brady, A. Lawrence-Douglas, M. G. Ramsay, N. J. Sircombe, P. Gillies, R. G. Evans, H. Schmitz, A. R. Bell, C. P. Ridgers, "Contemporary particle-in-cell approach to laser-plasma modelling," Plasma Phys. Control. Fusion 57, 113001 (2015).

4. N.G. Denisov, "On a Singularity of the Field on an Electromagnetic Wave Propagated in an Inhomogeneous Plasma," JETP 4, 544 (1957). 